\title{
Mamdani-Type Fuzzy-Based Adaptive Nonhomogeneous Synchronization
}

\author{
J. R. Pulido-Luna $\mathbb{D}^{1},{ }^{1}$ J. A. López-Rentería $\mathbb{D}^{2}{ }^{2}$ and N. R. Cazarez-Castro $\mathbb{D}^{1}$ \\ ${ }^{1}$ Departamento de Ingeniería Eléctrica y Electrónica, Tecnológico Nacional de México, Instituto Tecnológico de Tijuana, \\ Calzada Tecnológico S/N, Fracc, Tomás Aquino, Tijuana, Baja California, CP 22414, Mexico \\ ${ }^{2}$ CONACyT-Tecnológico Nacional de México, Instituto Tecnológico de Tijuana, Calzada Tecnológico S/N, Fracc, Tomás Aquino, \\ Tijuana, Baja California, CP 22414, Mexico \\ Correspondence should be addressed to J. A. López-Rentería; jorge.lopez@tectijuana.edu.mx and N. R. Cazarez-Castro; nohe@ \\ ieee.org
}

Received 26 March 2021; Accepted 28 July 2021; Published 24 August 2021

Academic Editor: Zakia Hammouch

Copyright (c) 2021 J. R. Pulido-Luna et al. This is an open access article distributed under the Creative Commons Attribution License, which permits unrestricted use, distribution, and reproduction in any medium, provided the original work is properly cited.

The aim of this work is the design of an adaptive controller based on Mamdani-type fuzzy inference systems. The input control is constructed with saturation functions' fuzzy-equivalents, which works as the adaptive scheme of the controller. This control law is designed to stabilize the error system to synchronize a pair of chaotic nonhomogeneous piecewise systems. Finally, an illustrative example as numerical evidence is developed.

\section{Introduction}

The synchronization phenomena among dynamical systems are a widely studied topic in the last decades due to the vast amount of applications in science and engineering [1-3]. In the related literature, dynamical systems and synchronization applications in many fields can be found, from biology $[4,5]$, mechanical systems [6-9], chemistry [10], physics $[11,12]$, fuzzy modeling [13-16] to secure communications [17-19], among many others. In general, it is said that a set of dynamical systems achieve synchronization if trajectories in each system approach a common trajectory.

Among the systems studied in synchronization, the ones that stand out are the chaotic systems; chaotic systems exhibit more complex dynamics, and they must satisfy the next conditions according to Devaney's definition of chaos [20]: (i) sensitive dependence to initial conditions, (ii) dense periodic orbits, and (iii) must be transitive. Many works consider the problem of chaos synchronization; in [21], the authors synchronize chaotic systems by linking them with common signals. Chua et al. [22] explore the synchronization phenomena in Chua's circuit, proven to be the simplest electronic circuit to exhibit chaotic behaviour; on the contrary, Femat and Solís-Perales [23] discuss several phenomena involved with chaos synchronization, and a feedback controller is implemented to illustrate such synchronization. In [24], chaos synchronization between two coupled chaotic dynamical systems is presented. Conditions for global asymptotic synchronization are presented and a new method for the analysis of the stability of the synchronization is reported, but different techniques and applications are still developed for this type of system in the last years. In [25], the authors present the design of a rule-based controller for a class of master-slave chaos synchronization, and unlike traditional methods, the control law obtained from this method has less maximum magnitude of the control signal and reduces the actuator saturation phenomenon in mechanical systems. AL-Azzawi and Aziz [26] present the synchronization between two nonhomogeneous hyperchaotic systems. A nonlinear control is used to achieve synchronization and also report a stability analysis of the error dynamics system using Lyapunov's second method and Cardano's method. In [27], higher-order adaptive PID controllers as a new generation of PID controllers for chaos 
synchronization is designed, and in [28], the authors study the collected dynamics of a $n$-coupled piecewise linear systems with different numbers of scrolls.

Along the synchronization schemes and controllers designed, the adaptive type has been proven useful depending on the application [29-32], and this kind of controller allows the optimization of the energy necessary to accomplish the synchronization between systems.

$\mathrm{Wu}$ et al. [29] study the synchronization of two chaotic systems which are not identical and use adaptive controllers to adjust the parameters of the systems such that the two systems will synchronize. In [25], the design of a rule-based fuzzy controller for a class of master-slave chaos synchronization is presented; however, the whole control action is substituted by the fuzzy controller, while Xi et al. propose an adaptive robust finite-time control method based on a global sliding surface for the synchronization of a class of chaotic systems in [32].

The main contribution of this work is the design of an adaptive synchronization scheme based on a Mamdani-type inference system, an equivalence with saturation functions is used, and the stability of the error system is proven using Lyapunov stability theory. Both, master and slave systems can be described in as many pieces as necessary. In order to provide evidence of energy optimization, a comparison between fixed gain and adaptive gain in the same synchronization scheme is reported in a satisfactory fashion.

The rest of this work is organized as follows. Section 2 presents the problem statement and the systems description. Section 3 introduces the basic concepts of synchronization and Mamdani fuzzy inference system (FIS). Section 4 presents the main results with an example as numeric evidence; finally, in Section 5, the conclusions are presented.

\section{Problem Statement}

Consider a classic master-slave synchronization scheme as

$$
\begin{aligned}
& \text { Master: } \dot{x}=f(x), \\
& \text { Slave: } \dot{y}=g(y)+u,
\end{aligned}
$$

where both the master and slave system are piecewise switching systems [33-36] of the form

$$
\dot{z}=\mathrm{Az}+B
$$

with

$$
B=\left\{\begin{array}{ccc}
B_{1} & \text { if } & z \in \mathscr{D}_{1} \\
B_{2} & \text { if } & z \in \mathscr{D}_{2} \\
\vdots & \vdots & \vdots \\
B_{j} & \text { if } & z \in \mathscr{D}_{j}
\end{array},\right.
$$

that undergo chaotic behaviour, where $x, y \in \mathbb{R}^{3}$ are the state variables, $B_{j}=\left(b_{j 1}, b_{j 2}, b_{j 3}\right)^{T}$, which are the switching laws of the piecewise chaotic systems, and $u \in \mathbb{R}^{3}$ is the actuator in charge of achieve the synchronization of the slave over the master. Moreover, the domains $\mathscr{D}_{j}$ satisfy $\mathbb{R}^{3}=$ $\cup_{j=1}^{m} \mathscr{D}_{j}$ and $\cap \cap_{j=1}^{m} \mathscr{D}_{j}=\varnothing$ for $m>1$. The objective of this work is to design the controller $u$ as an adaptive controller of the form

$$
u=\left\{\begin{array}{ccc}
u_{1} & \text { if } & y \in \mathscr{E}_{1} \\
u_{2} & \text { if } & y \in \mathscr{E}_{2} \\
\vdots & \vdots & \vdots \\
u_{k} & \text { if } & y \in \mathscr{E}_{k}
\end{array}\right.
$$

for $k=1,2, \ldots, n$, using a Mamdani-based fuzzy inference system for each $u_{k}$ to synchronize the master and slave systems, optimizing the energy usage for the control action. Consequently, there are three major objectives to develop in this work:

(1) The equivalence relation between the fuzzy-based controller and the saturation functions

(2) Achieve adaptive synchronization between systems

(3) Ensure asymptotic stability of the error system via Lyapunov's theory

\section{Preliminaries}

In this section, the basic concepts of synchronization and fuzzy inference system design will be presented; this concept will be convenient to understand how it is possible to achieve the principal objective of this work.

3.1. Synchronization Scheme. Consider the master-slave synchronization scheme defined in [37] as

$$
\begin{aligned}
& \text { Master: } \dot{x}=f(x), \\
& \text { Slave: } \dot{y}=g(y)+u,
\end{aligned}
$$

with $x, y \in \mathbb{R}^{n}$ as the system states and

$$
u=-P e+f(x)-g(y),
$$

where $P=\operatorname{diag}\left\{p_{1}^{2}, p_{2}^{2}, p_{3}^{2}\right\}$. The synchronization is achieved when the master and slave system synchronize, i.e., when the synchronization objective,

$$
\lim _{t \rightarrow \infty}\|y(t)-x(t)\|=0
$$

is reached.

Definition 1 (see [3]). A system of the form $\dot{x}=f(x)$ is called master system if its flow $x(t)$ is independent. A system of the form $\dot{y}=g(y)+u$ is called slave system of the master system if its flow $y(t)$ is constrained by the flow of the master system.

From the previous definition, a slave system is constrained by a master system via a specific condition, which means that a slave system will be controlled towards a master system under a specific control law. This phenomenon is called the synchronization of the slave and master systems under such a specific condition $[3,38]$. 
3.2. Fuzzy Inference System Design. The design of the fuzzy inference systems considers an IF-THEN fuzzy rules based on the form [39]

$$
R_{i} \text { : IF } e \text { is } M_{i}, \quad \text { THEN } p \text { is } H_{i},
$$

where $e$ is the error between two states, and the input of the membership functions is

$$
\begin{aligned}
& M_{-1}(e)= \begin{cases}1, & \text { if } e<\Phi_{-1}, \\
\frac{1}{\Phi_{-1}} e, & \text { if } \Phi_{-1} \leq e \leq \Phi_{0}, \\
0, & \text { if } e>\Phi_{0},\end{cases} \\
& M_{0}(e)= \begin{cases}-\frac{1}{\Phi_{-1}} e+1, & \text { if } \Phi_{-1} \leq e<\Phi_{0}, \\
-\frac{1}{\Phi_{1}} e+1, & \text { if } \Phi_{0} \leq e \leq \Phi_{1}, \\
0, & \text { elsewhere, }\end{cases} \\
& M_{1}(e)= \begin{cases}0, & \text { if } e<\Phi_{0}, \\
\frac{1}{\Phi_{1}} e, & \text { if } \Phi_{0} \leq e \leq \Phi_{1}, \\
1, & \text { if } e>\Phi_{1},\end{cases}
\end{aligned}
$$

with $\Phi_{-1}=-\Phi_{1}$, while the output membership functions are singletons, defined as

$$
\begin{aligned}
& H_{-1}(p)= \begin{cases}1, & \text { if } p=\Omega_{-1}, \\
0, & \text { elsewhere }\end{cases} \\
& H_{0}(p)= \begin{cases}1, & \text { if } p=\Omega_{0} \\
0, & \text { elsewhere }\end{cases} \\
& H_{1}(p)= \begin{cases}1, & \text { if } p=\Omega_{1}, \\
0, & \text { elsewhere }\end{cases}
\end{aligned}
$$

The input and output membership functions are depicted in Figure 1. Now, consider the Mamdani-type fuzzy inference system, with product in the antecedent and center of average as the defuzzifier method. Then, the fuzzy inference system is written as

$$
p(e)=\sum_{i=-1}^{1}\left\{\frac{M_{i}(e)}{\sum_{j=-1}^{1} M_{j}(e)}\right\} \Omega_{i}=\sum_{i=-1}^{1} X_{i}(e) \Omega_{i},
$$

where $\Omega_{i}$ refers the crisp value of the output regarding of $e$. The function $X_{i}(e)$ must satisfy the following conditions $[40,41]$ : (i) $X_{i}(e)$ is a locally Lipschitz continuous and bounded function

(ii) $X_{i}(0)=0$

(iii) $X_{i}(e)=X_{i}(-e)$

The designed rule base matrix is given in Table 1.

Only two rules are fired at the same time [41, 42], for any value of $e$. Moreover, it satisfies

$$
\sum_{i=-1}^{1} X_{i}(e)=1,
$$

which is a linear convex combination.

\section{Main Results}

Consider the synchronization error as $e=y-x$. The error dynamics are defined as

$$
\dot{e}=g(y)-f(x)+u,
$$

where the controller $u$ is

$$
u=-P e+f(x)-g(y),
$$

with $P=\operatorname{diag}\left\{p_{1}^{2}, p_{2}^{2}, p_{3}^{2}\right\}$. In the following, given the sectorial functions provided from the FIS (15), it is necessary to consider the matrix of adapting $P=\operatorname{diag}\left\{p_{1}^{2}\left(e_{1}\right)\right.$, $\left.p_{2}^{2}\left(e_{2}\right), p_{3}^{2}\left(e_{3}\right)\right\}$. Then, we can establish the following result.

Lemma 1. The controller $u=-P e+f(x)-g(y)$ can be expressed as

$$
u=-\left(\begin{array}{l}
\left(\Omega_{1}^{1} \operatorname{sat}\left(\frac{e_{1}}{\Phi_{1}^{1}}\right)\right)^{2} e_{1} \\
\left(\Omega_{1}^{2} \operatorname{sat}\left(\frac{e_{2}}{\Phi_{1}^{2}}\right)\right)^{2} e_{2} \\
\left(\Omega_{1}^{3} \operatorname{sat}\left(\frac{e_{3}}{\Phi_{1}^{3}}\right)\right)^{2} e_{3}
\end{array}\right)+f(x)-g(y) .
$$

Proof. For each $l=1,2,3$, it is possible to rewrite (15) as

$$
p_{l}\left(e_{l}\right)= \begin{cases}\frac{\Omega_{1}^{l}}{\Phi_{1}^{l}} e_{l}, & \text { if }\left|e_{l}\right| \leq \Phi_{1}^{l}, \\ \Omega_{1}^{l}, & \text { if }\left|e_{l}\right|>\Phi_{1}^{l} .\end{cases}
$$

Thus, each FIS can be written as a piecewise linear function as follows: 


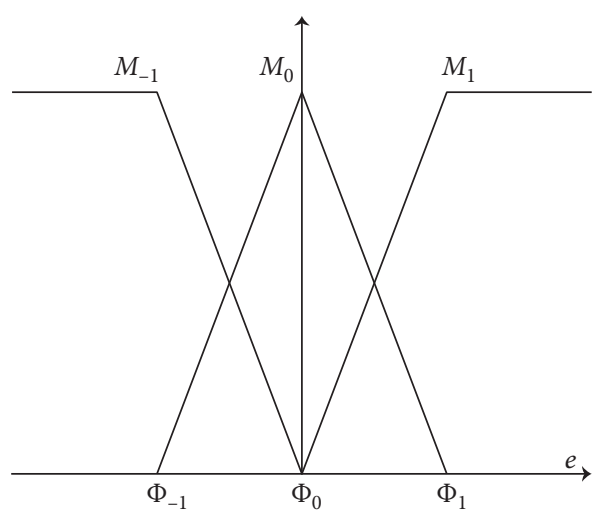

(a)

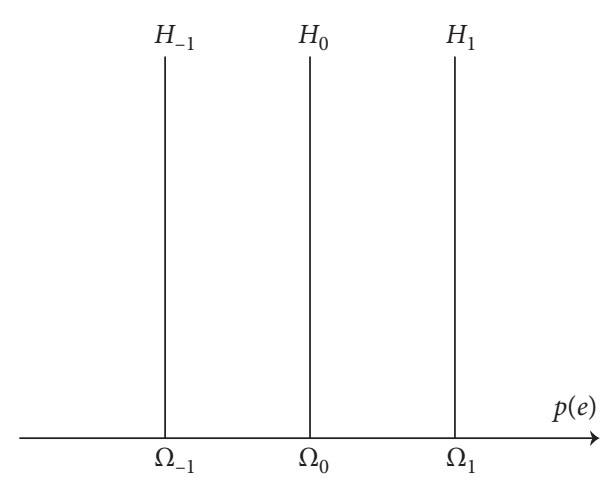

(b)

FIgUre 1: Membership functions: (a) input; (b) output.

Table 1: Fuzzy rule base.

\begin{tabular}{lc}
\hline Input $(e)$ & Output $(p(e))$ \\
\hline$M_{-1}$ & $H_{-1}$ \\
$M_{0}$ & $H_{0}$ \\
$M_{1}$ & $H_{1}$ \\
\hline
\end{tabular}

$$
\begin{aligned}
p_{l}\left(e_{l}\right)=\Omega_{1}^{l} \begin{cases}\frac{e_{l}}{\Phi_{1}^{l},} & \text { if }\left|e_{l}\right| \leq \Phi_{1}^{l}, \\
1, & \text { if }\left|e_{l}\right|>\Phi_{1}^{l},\end{cases} \\
=\Omega_{1}^{l} \begin{cases}\frac{e_{l}}{\Phi_{1}^{l}}, & \text { if } \frac{e_{l}}{\Phi_{1}^{l}} \leq 1, \\
1, & \text { if } \frac{e_{l}}{\Phi_{1}^{l}}>1,\end{cases} \\
=\Omega_{1}^{l} \operatorname{sat}\left(\frac{e_{l}}{\Phi_{1}^{l}}\right) .
\end{aligned}
$$

Substituting (18) in each $p_{l}$ of (15), it is possible to rewrite $u$ as

$$
u=-\left(\begin{array}{l}
\left(\Omega_{1}^{1} \operatorname{sat}\left(\frac{e_{1}}{\Phi_{1}^{1}}\right)\right)^{2} e_{1} \\
\left(\Omega_{1}^{2} \operatorname{sat}\left(\frac{e_{2}}{\Phi_{1}^{2}}\right)\right)^{2} e_{2} \\
\left(\Omega_{1}^{3} \operatorname{sat}\left(\frac{e_{3}}{\Phi_{1}^{3}}\right)\right)^{2} e_{3}
\end{array}\right)+f(x)-g(y),
$$

\footnotetext{
as previously stated.
}

Once the piecewise controller is described, whose fuzzy component is the candidate to work as the adaptive scheme, in the following result, we formalize this assertion.

Theorem 1. The synchronization scheme formed by (1) and (2) achieves synchronization under the adaptive controller (16). Thus, the error system (14) is asymptotically stable.

Proof. Consider the quadratic Lyapunov candidate function:

$$
V(e)=\frac{1}{2} e^{T} P e
$$

From Lemma 1, it is known that

$$
P e=\left(\begin{array}{l}
\left(\Omega_{1}^{1} \operatorname{sat}\left(\frac{e_{1}}{\Phi_{1}}\right)\right)^{2} e_{1} \\
\left(\Omega_{1}^{2} \operatorname{sat}\left(\frac{e_{2}}{\Phi_{1}}\right)\right)^{2} e_{2} \\
\left(\Omega_{1}^{3} \operatorname{sat}\left(\frac{e_{3}}{\Phi_{1}}\right)\right)^{2} e_{3}
\end{array}\right) .
$$

The time derivative of (20) is computed as

$$
\begin{aligned}
\dot{V}(e) & =\frac{1}{2}\left(\frac{\partial V}{\partial e_{1}}+\frac{\partial V}{\partial e_{2}}+\frac{\partial V}{\partial e_{3}}\right), \\
& =e^{T} \dot{e}, \\
& =e^{T}(g(y)-f(x)+u) .
\end{aligned}
$$

Substituting the adaptive control law (16) in (22), 


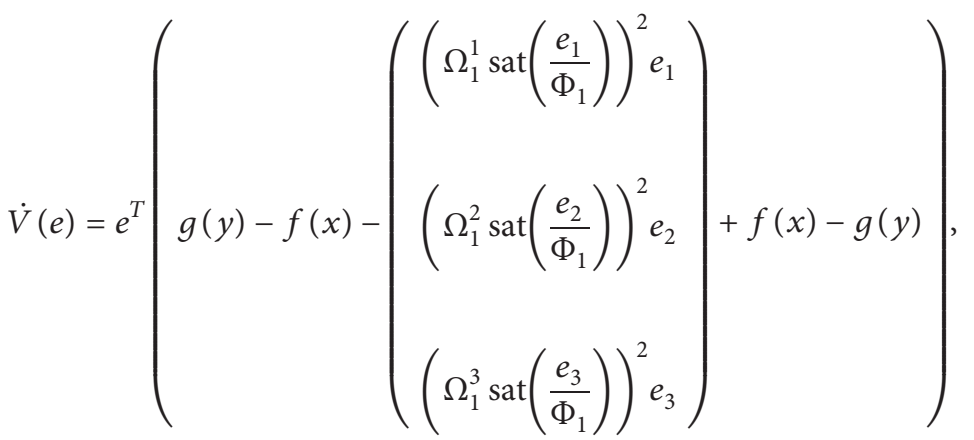

$$
\begin{aligned}
& =-e^{T}\left(\begin{array}{l}
\left(\Omega_{1}^{1} \operatorname{sat}\left(\frac{e_{1}}{\Phi_{1}}\right)\right)^{2} e_{1} \\
\left(\Omega_{1}^{2} \operatorname{sat}\left(\frac{e_{2}}{\Phi_{1}}\right)\right)^{2} e_{2} \\
\left(\Omega_{1}^{3} \operatorname{sat}\left(\frac{e_{3}}{\Phi_{1}}\right)\right)^{2} e_{3}
\end{array}\right) \\
& =-e^{T}\left(\begin{array}{ccc}
\left(\Omega_{1}^{1} \operatorname{sat}\left(\frac{e_{1}}{\Phi_{1}^{1}}\right)\right)^{2} & 0 & 0 \\
0 & \left(\Omega_{1}^{2} \operatorname{sat}\left(\frac{e_{2}}{\Phi_{1}^{2}}\right)\right)^{2} & 0 \\
0 & 0 & \left(\Omega_{1}^{3} \operatorname{sat}\left(\frac{e_{3}}{\Phi_{1}^{3}}\right)\right)^{2}
\end{array}\right) e, \\
& =-e^{T} P e<0,
\end{aligned}
$$

which ensures the negativeness of (22) and consequently the asymptotic stability of the error system.

The following example illustrates the effectiveness and performance of the controller design, which comes to corroborate what we claim and prove.

Example 1. According to [43], we synthesized the following master system:

$$
\dot{x}=\left(\begin{array}{ccc}
0 & 1 & 0 \\
0 & 0 & 1 \\
-a_{1} & -a_{2} & -a_{3}
\end{array}\right) x+\left(\begin{array}{c}
-\beta_{1} \\
-\beta_{2} \\
a_{1} \beta_{3}+a_{2} \beta_{1}+a_{3} \beta_{2}
\end{array}\right) \text {, }
$$

where $a_{1}=3 / 2, a_{2}=1, a_{3}=1$, and

$$
\begin{aligned}
& \beta_{1}= \begin{cases}5 & \text { if } x_{2} \geq 0, \\
-5 & \text { if } x_{2}<0,\end{cases} \\
& \beta_{2}= \begin{cases}14 & \text { if } x_{3} \geq 7, \\
0 & \text { if } x_{3}<7,\end{cases} \\
& \beta_{3}= \begin{cases}4 & \text { if } x_{1} \geq 0, \\
-4 & \text { if } x_{1}<0 .\end{cases}
\end{aligned}
$$

In Figure 2, the 3D phase portrait plot of system (24) is depicted under initial conditions $x_{0}=(0,4,10)^{T}$, and we can see that the master system presents a 8 -scroll chaotic attractor in three different directions. The projections in the planes $\left(x_{1}, x_{2}\right),\left(x_{1}, x_{3}\right)$, and $\left(x_{2}, x_{3}\right)$ for the system are shown in Figure 3.

Now, following the same methodology used to design the master system, consider the slave system as 


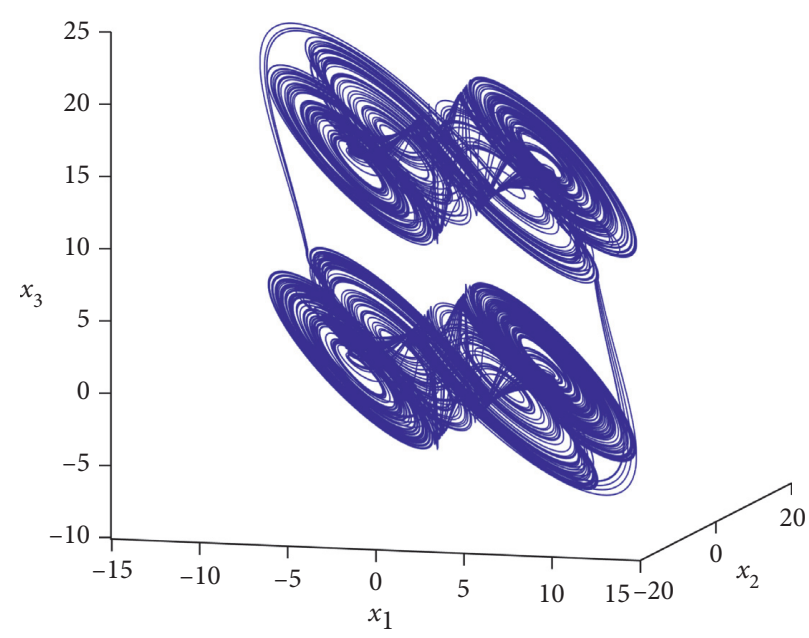

Figure 2: 3D phase portrait of the master chaotic system.

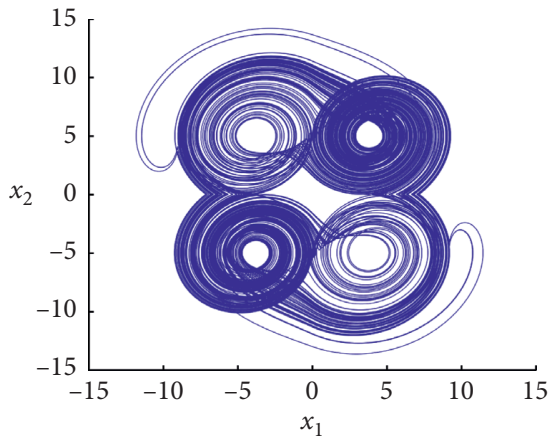

(a)

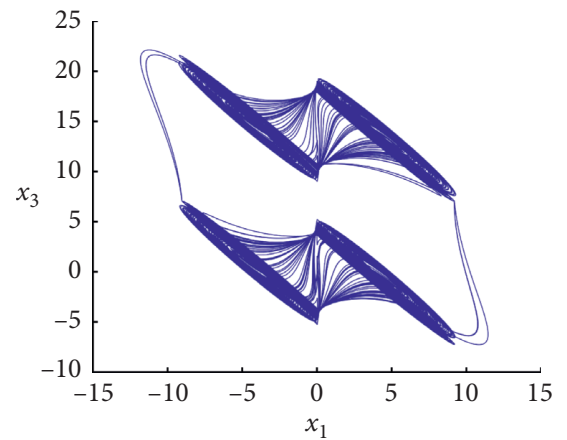

(b)

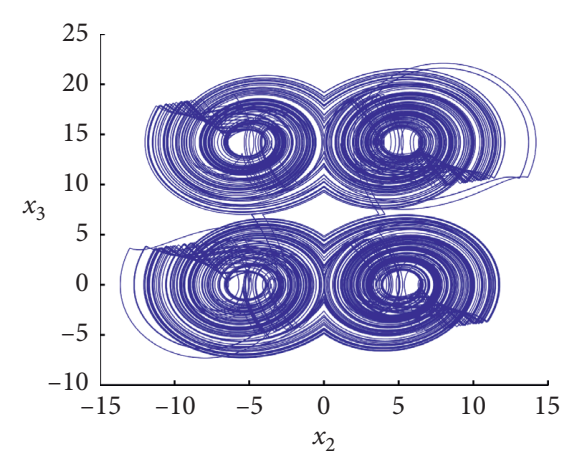

(c)

FIGURE 3: Plane projections for the master system: (a) $\left(x_{1}, x_{2}\right)$; (b) $\left(x_{1}, x_{3}\right)$; (c) $\left(x_{2}, x_{3}\right)$.

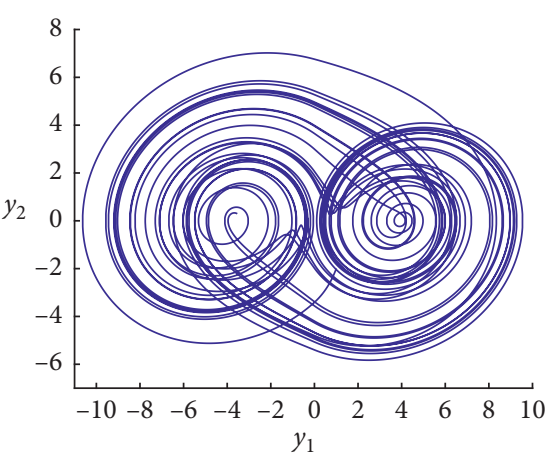

(a)

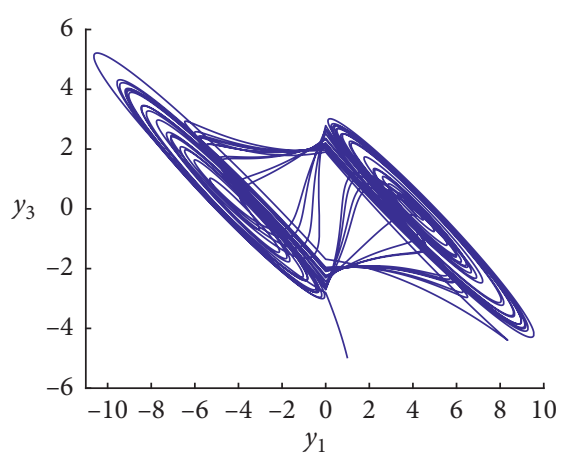

(b)

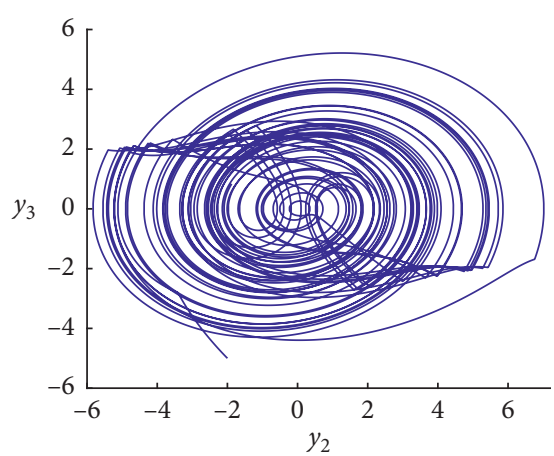

(c)

FIGURE 4: Slave system plane projections: (a) $\left(y_{1}, y_{2}\right)$; (b) $\left(y_{1}, y_{3}\right)$; (c) $\left(y_{2}, y_{3}\right)$. 


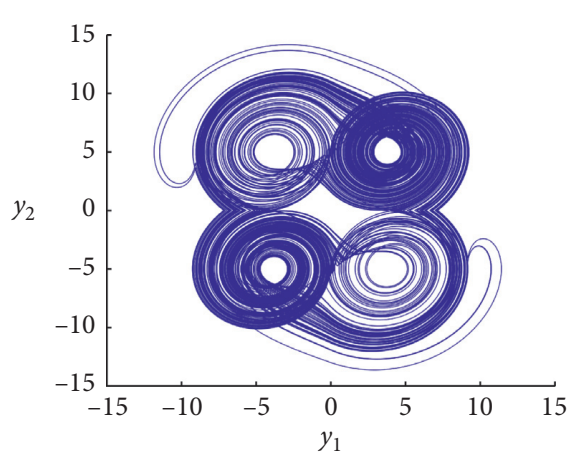

(a)

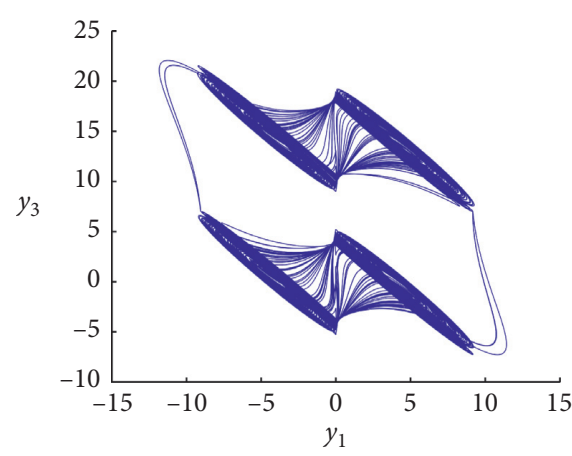

(b)

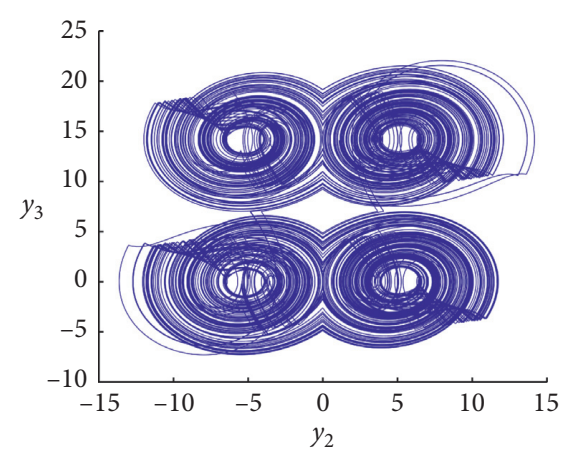

(c)

FigurE 5: Synchronized slave system plane projections: (a) $\left(y_{1}, y_{2}\right)$; (b) $\left(y_{1}, y_{3}\right)$; (c) $\left(y_{2}, y_{3}\right)$.

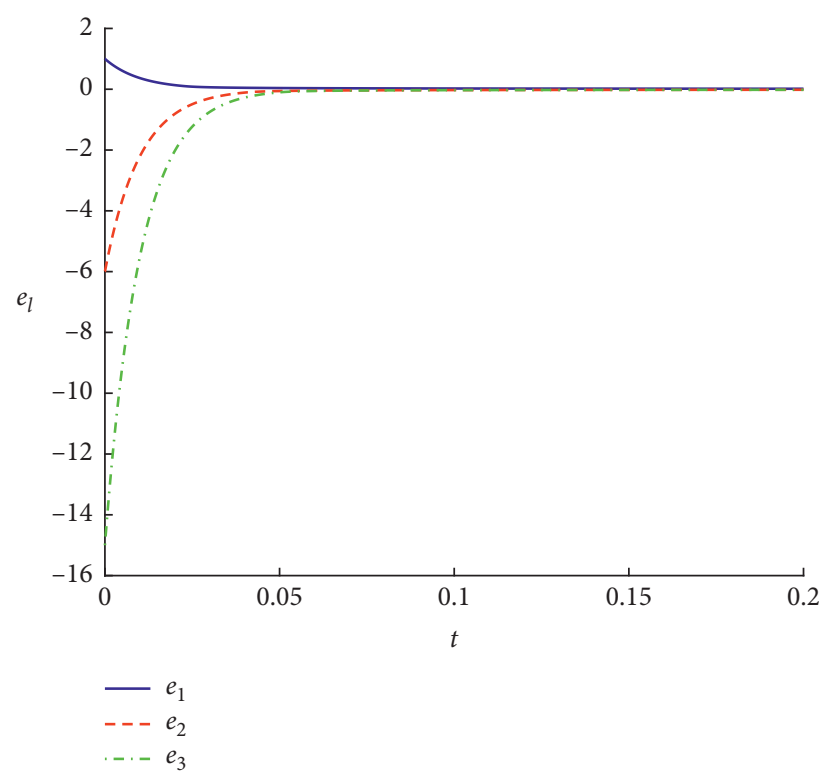

FIgURE 6: Error dynamics.
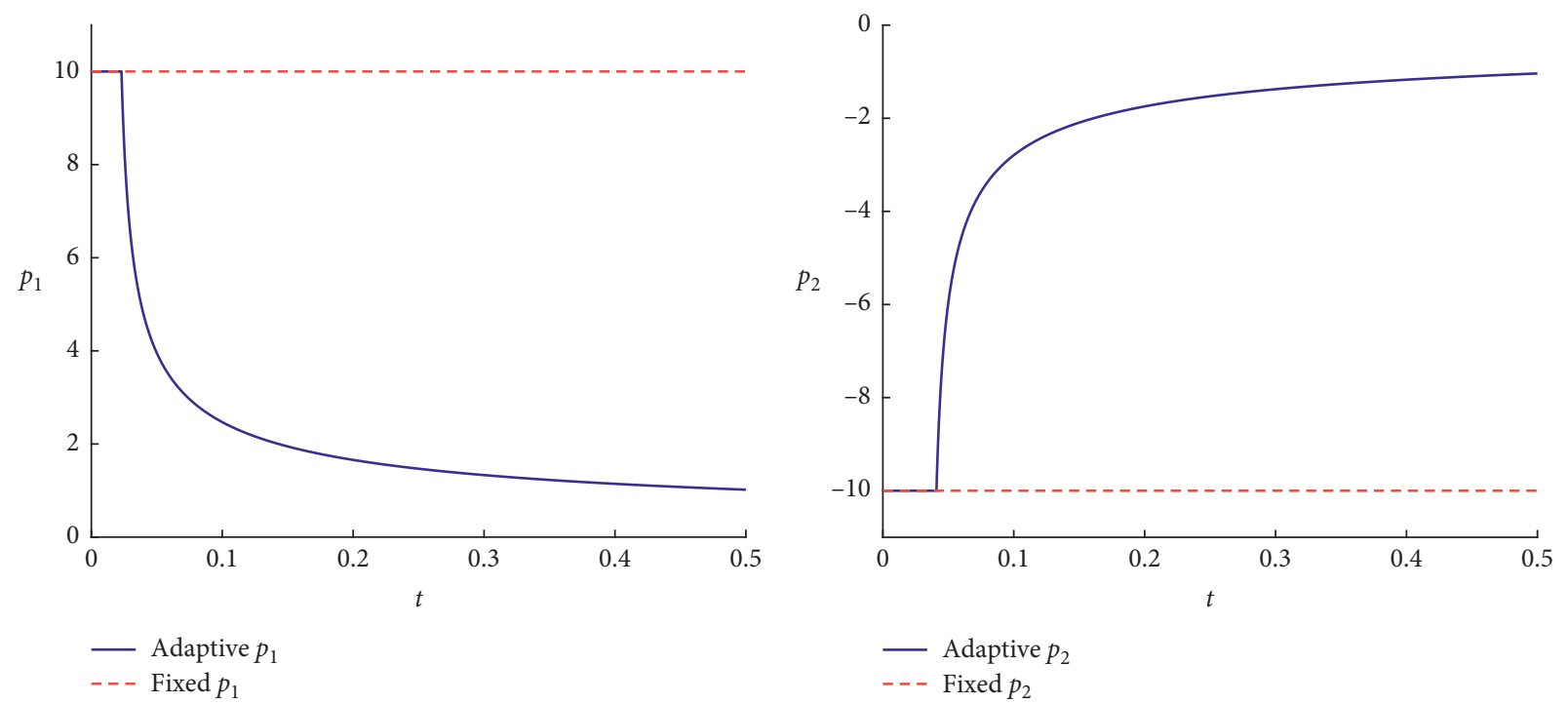

(a)

Figure 7: Continued. 


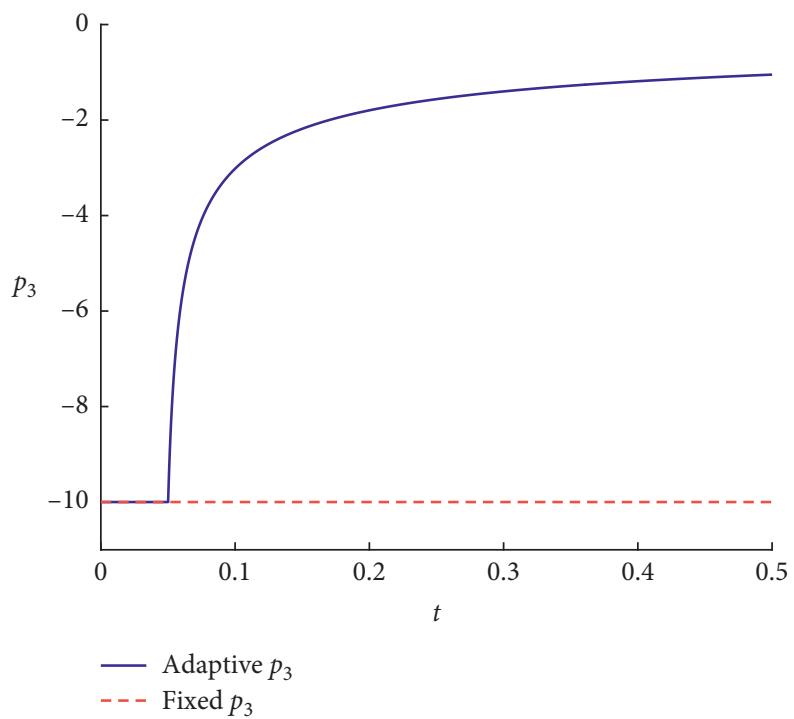

(c)

Figure 7: Evolution of $p_{l}\left(e_{l}\right)$ trough time: (a) $p_{1}\left(e_{1}\right)$; (b) $p_{2}\left(e_{2}\right)$; (c) $p_{3}\left(e_{3}\right)$.

$$
\dot{y}=\left(\begin{array}{rrr}
0 & 1 & 0 \\
0 & 0 & 1 \\
-\widetilde{a}_{1} & -\widetilde{a}_{2} & -\widetilde{a}_{3}
\end{array}\right) y+\left(\begin{array}{c}
0 \\
0 \\
\widetilde{a}_{1} \widetilde{\beta}_{3}
\end{array}\right)+u,
$$

with $\tilde{a}_{i}=3 / 5$, for $i=1,2,3$, and

$$
\widetilde{\beta}_{3}= \begin{cases}4 & \text { if } y_{1} \geq 0 \\ -4 & \text { if } y_{1}<0\end{cases}
$$

Unlike the master system, the slave system only presents a double scroll chaotic attractor by taking the initial condition point $y_{0}=(1,-2,-5)^{T}$, whose projections in the planes $\left(y_{1}, y_{2}\right),\left(y_{1}, y_{3}\right)$, and $\left(y_{2}, y_{3}\right)$ for the slave system, when $u=(0,0,0)^{T}$, are shown in Figure 4 .

The control law $u$ is designed according to Lemma 1 , and its explicit expression is given by

$$
\begin{aligned}
u= & -P e+f(x)-g(y), \\
= & -\left(\begin{array}{l}
\left(\Omega_{1}^{1} \operatorname{sat}\left(\frac{e_{1}}{\Phi_{1}^{1}}\right)\right)^{2} e_{1} \\
\left(\Omega_{1}^{2} \operatorname{sat}\left(\frac{e_{2}}{\Phi_{1}^{2}}\right)\right)^{2} e_{2} \\
\left(\Omega_{1}^{3} \operatorname{sat}\left(\frac{e_{3}}{\Phi_{1}^{3}}\right)\right)^{2} e_{3}
\end{array}\right)+\left(\begin{array}{ccc}
0 & 1 & 0 \\
-\beta_{1} & 0 & 1 \\
0 & \\
-a_{1} & -a_{2} & -a_{3}
\end{array}\right) x, \\
& +\left(\begin{array}{c}
\beta_{2} \\
a_{1} \beta_{3}+a_{2} \beta_{1}+a_{3} \beta_{2}
\end{array}\right)-\left(\begin{array}{ccc}
0 & 1 & 0 \\
0 & 0 & 1 \\
-\tilde{a}_{1} & -\tilde{a}_{2} & -\tilde{a}_{3}
\end{array}\right) y-\left(\begin{array}{c}
0 \\
0 \\
\tilde{a}_{1} \tilde{\beta}_{3}
\end{array}\right),
\end{aligned}
$$




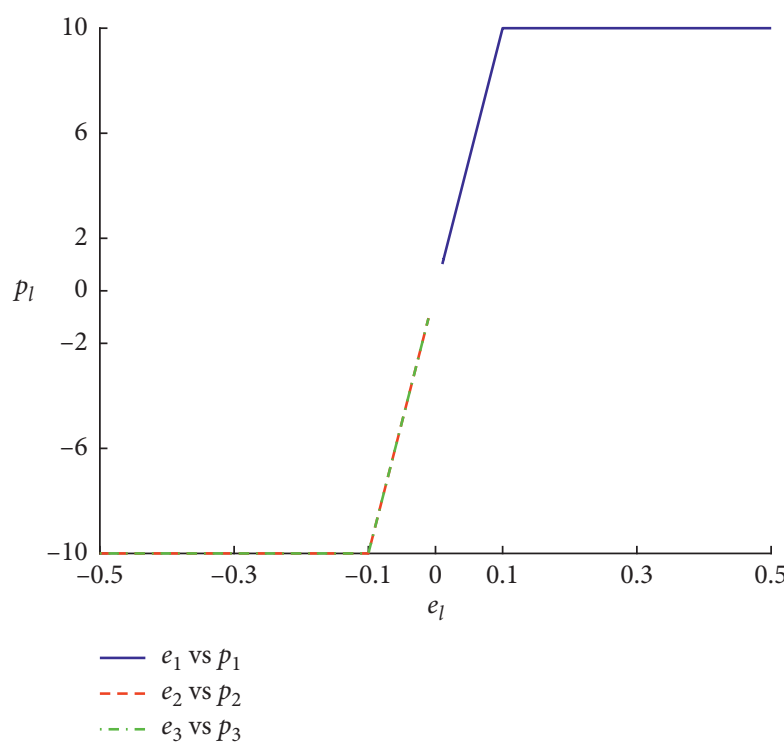

(a)

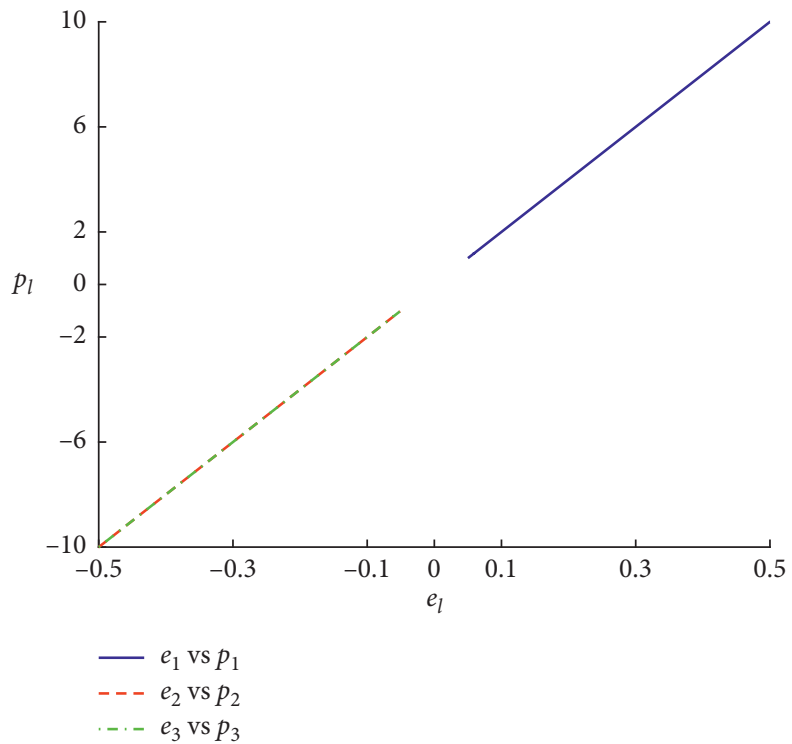

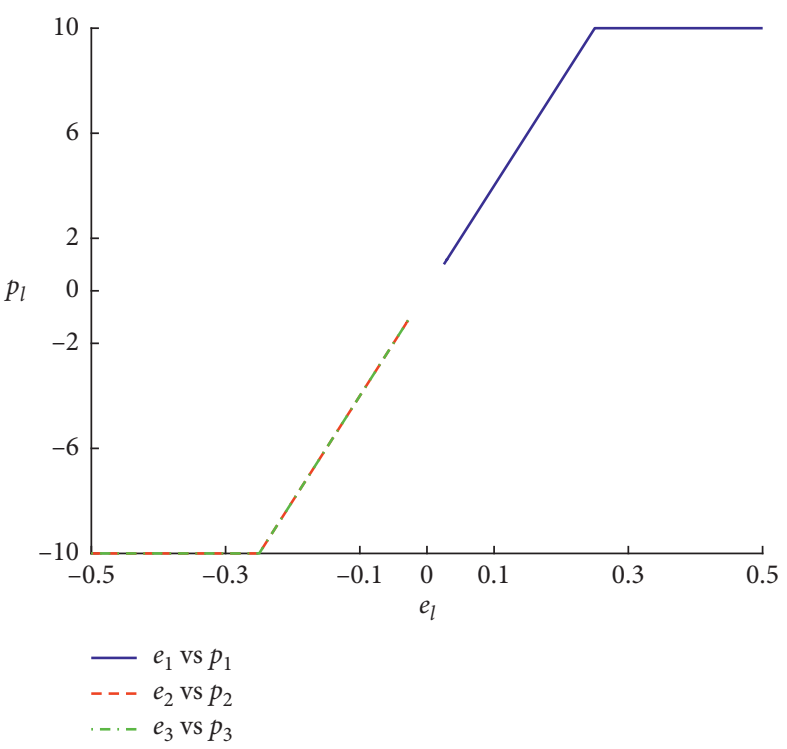

(b)

(c)

Figure 8: Comparative between the adaptation domains for (a) $\Phi_{1}^{l}=0.1$; (b) $\Phi_{1}^{l}=0.25$; (c) $\Phi_{1}^{l}=0.5$.

where $\Omega_{1}^{l}=10$ and $\Phi_{1}^{l}=1 / 10$, for $l=1,2,3$. The projections in the planes $\left(y_{1}, y_{2}\right),\left(y_{1}, y_{3}\right)$, and $\left(y_{2}, y_{3}\right)$ for the synchronized slave system are shown in Figure 5. It is clear that the slave system now exhibits the trajectories of the master system and went from a double scroll chaotic attractor to a 8scroll chaotic attractor.

The errors between the master and slave systems are depicted in Figure 6, and these errors tend asymptotically to the origin as intended.

4.1. Energy Optimization. One of the advantages of using the adaptive synchronization proposed in this work is the optimization of the energy used to induce the dynamics of the master system in the slave system. Comparing the magnitude of $p_{l}\left(e_{l}\right), l=1,2,3$, against the magnitude of a fixed value shows clearly that the energy consumption is vastly improved.

At first, high values of $p_{l}\left(e_{l}\right)$ are injected in the fuzzy actuator in order that the error $e_{l}$ reaches its following adaptive domain interval $\mathscr{A}_{l}=\left(-\Phi_{1}^{l}, \Phi_{1}^{l}\right)$, and then, the value of $p_{l}\left(e_{l}\right)$ decays until the error reaches the next interval of adaptation. Hence, the power stills decaying whilst the error system tends to zero.

Figure $7(\mathrm{a})$ shows the behaviour of the adaptive $p_{1}\left(e_{1}\right)$ (blue line) and the behaviour of a fixed not adaptive value of $p_{1}$ (dotted red line). This behaviour is also presented among the adaptives $p_{2}\left(e_{2}\right)$ and $p_{3}\left(e_{3}\right)$ (blue lines) and their respective fixed no adaptive values $p_{2}$ and $p_{3}$ (in red dots), depicted in Figures 7(b) and 7(c), respectively. 
The energy optimization can be modified according the application where it is planned to be used by modifying the adapting interval $\mathscr{A}_{l}$ of each $p_{l}\left(e_{l}\right)$. This can be better appreciated in Figures $8(\mathrm{a})-8(\mathrm{c})$.

\section{Conclusions}

An adaptive synchronization scheme was provided using as adaptation law a Mamdani-type fuzzy inference system. An equivalence is given to express the fuzzy inference system as saturation functions and evidence is provided to ensure the stability of the error system between the master and the slave systems.

\section{Data Availability}

All underlying data that support results can be found in Tecnológico Nacional de México, Instituto Tecnológico de Tijuana, Calzada Tecnológico S/N, Tijuan, Baja California, México.

\section{Conflicts of Interest}

The authors declare that they have no conflicts of interest.

\section{Acknowledgments}

This research was supported by CONACyT with project no. A1-S-32341 and Tecnológico Nacional de México with project nos. 5564.19-P, 8085.20-P, and 11122.21-P. J. R. Pulido-Luna wants to thank CONACyT for the DSc scholarship.

\section{References}

[1] A. Pikovsky, M. Rosenblum, and J. K. Synchronization, “A universal concept in nonlinear sciences," in The Edinburgh Building, Cambridge University Press, Cambridge, UK, 1st edition, 2001.

[2] S. Boccaletti, "The synchronized dynamics of complex systems," Monograph Series on Nonlinear Science and Complexity, vol. 6, 2008.

[3] A. C. J. Luo, "A theory for synchronization of dynamical systems," Communications in Nonlinear Science and $\mathrm{Nu}$ merical Simulation, vol. 14, no. 5, pp. 1901-1951, 2009.

[4] Z. Qu, "Chaos in the genesis and maintenance of cardiac arrhythmias," Progress in Biophysics and Molecular Biology, vol. 105, no. 3, pp. 247-257, 2011.

[5] L. J. Ontañón-García and E. Campos-Cantón, "Discrete coupling and synchronization in the insulin release in the mathematical model of the $\beta$ Cells," Discrete Dynamics in Nature and Society, vol. 2013, Article ID 427050, 7 pages, 2013.

[6] P. J. Prieto, N. R. Cazarez-Castro, L. T. Aguilar, and S. L. Cardenas-Maciel, "Chattering existence and attenuation in fuzzy-based sliding mode control," Engineering Applications of Artificial Intelligence, vol. 61, pp. 152-160, 2017.

[7] P. J. Prieto-Entenza, L. T. Aguilar, S. L. Cardenas-Maciel, J. A. Lopez-Renteria, and N. R. Cazarezcastro, "Stability analysis for mamdani-type integral fuzzy-based sliding mode control of systems under persistent disturbances," IEEE Transactions on Fuzzy Systems, p. 1. In press, 2021.
[8] L. Herrera-Garcia, N. R. Cazarez-Castro, S. L. CardenasMaciel, J. A. Lopez-Renteria, and L. T. Aguilar, "Self-excited periodic motion in underactuated mechanical systems using two-fuzzy inference system," Fuzzy Sets and Systems, 2021, In press.

[9] J. A. Lopez-Renteria, L. Herrera-Garcia, S. L. CardenasMaciel, L. T. Aguilar, and N. R. Cazarezcastro, "Self-sustaining oscillations with an internal two-fuzzy inference system based on the poincar-bendixson method," IEEE Transactions on Fuzzy Systems, p. 1. In press, 2021.

[10] S. Vaidyanathan, "Adaptive synchronization of chemical chaotic reactors," International Journal of ChemTech Research, vol. 8, no. 2, pp. 612-621, 2015.

[11] B. A. Idowu and U. E. Vincent, "Synchronization and stabilization of chaotic dynamics in a quasi-1D bose-einstein condensate," Journal of Chaos, vol. 2013, Article ID 723581, 8 pages, 2013.

[12] G. Yuan, X. Zhang, and Z. Wang, "Generation and synchronization of feedbackinduced chaos in semiconductor ring lasers by injectionlocking," Optik, vol. 125.8, Article ID 19501953, 2014.

[13] N. R. Cazarez-Castro, S. L. Cardenas-Maciel, M. OdremanVera, G. Valencia-Palomo, and C. Leal-Ramrez, "Modeling PD closed-loop control problems with fuzzy differential equations," Automatika, vol. 57, no. 4, pp. 960-967, 2016.

[14] N. R. Cazarez-Castro, M. Odreman-Vera, S. L. CardenasMaciel, H. Echavarria-Heras, and C. Leal-Ramirez, "Fuzzy differential equations as a tool for teaching uncertainty in engineering and science," Computación y Sistemas, vol. 22, no. 2, pp. 439-449, 2018.

[15] M. Arfan, K. Shah, T. Abdeljawad, and Z. Hammouch, "An efficient tool for solving two-dimensional fuzzy fractionalordered heat equation," Numerical Methods for Partial Differential Equations, vol. 37, no. 2, pp. 1407-1418, 2020.

[16] A. Ullah, Z. Ullah, T. Abdeljawad, Z. Hammouch, and K. Shah, "A hybrid method for solving fuzzy Volterra integral equations of separable type kernels," Journal of King Saud University-Science, vol. 33, no. 1, Article ID 101246, 2021.

[17] M. C. Soriano, I. Fischer, C. R. Mirasso, R. M. Nguimdo, and G. Van der Sande, "Encryption key distribution via chaos synchronization," Scientific Reports, vol. 7, no. 1, Article ID 43428, 2017.

[18] O. Guillén-Fernández, J. de Jesus Rangel-Magdaleno, A. Melendez-Cano, J. C. Nuñez-Perez, and L. G. de la Fraga, "FPGA implementation of chaotic oscillators, their synchronization, and application to secure communications," in Recent Advances in Chaotic Systems and Synchronization, pp. 301-328, Academic Press, Cambridge, MA, USA, 2019.

[19] S. Moon, J.-J. Baik, and J. M. Seo, "Chaos synchronization in generalized Lorenz systems and an application to image encryption," Communications in Nonlinear Science and Numerical Simulation, vol. 96, Article ID 105708, 2021.

[20] R. L. Devaney, An Introduction to Chaotic Dynamical Systems, Westview Press, Boulder, CO, USA, 2nd edition, 2003.

[21] L. M. Pecora and T. L. Carroll, "Synchronization in chaotic systems,” Physical Review Letters, vol. 64, no. 8, Article ID 821824, 1990.

[22] L. O. Chua, L. Kocarev, K. Eckert, and M. Itoh, "Experimental chaos synchronization in chua's circuit," International Journal of Bifurcation and Chaos, vol. 2, no. 3, pp. 705-708, 1992.

[23] R. Femat and G. Solís-Perales, "On the chaos synchronization phenomena," Physics Letters A, vol. 262, no. 1, Article ID 5060, 1999. 
[24] J. Lü, T. Zhou, and S. Zhang, "Chaos synchronization between linearly coupled chaotic systems," Chaos, Solitons Fractals, vol. 14, no. 4, Article ID 529541, 2002.

[25] H.-T. Yau and C.-S. Shieh, "Chaos synchronization using fuzzy logic controller," Nonlinear Analysis: Real World Applications, vol. 9, no. 4, pp. 1800-1810, 2008.

[26] S. F. AL-Azzawi and M. M. Aziz, "Chaos synchronization of nonlinear dynamical systems via a novel analytical approach," Alexandria Engineering Journal, vol. 57, no. 4, pp. 3493-3500, 2018.

[27] M. M. Zirkohi and S. Khorashadizadeh, "Chaos synchronization using higher-order adaptive PID controller," AEU-International Journal of Electronics and Communications, vol. 94, pp. 157-167, 2018.

[28] A. Anzo-Hernández, E. Campos-Cantón, and M. Nicol, "Itinerary synchronization between PWL systems coupled with unidirectional links," Communications in Nonlinear Science and Numerical Simulation, vol. 70, pp. 102-124, 2019.

[29] C. W. Wu, T. Yang, and L. O. Chua, "On adaptive synchronization and control of nonlinear dynamical systems," International Journal of Bifurcation and Chaos, vol. 6, no. 3, pp. 455-471, 1996.

[30] T.-L. Liao and S.-H. Tsai, "Adaptive synchronization of chaotic systems and its application to secure communications," Chaos, Solitons \& Fractals, vol. 11, no. 9, pp. 1387-1396, 2000.

[31] H. Zhang, W. Huang, Z. Wang, and T. Chai, “Adaptive synchronization between two different chaotic systems with unknown parameters," Physics Letters A, vol. 350, no. 5-6, pp. 363-366, 2006.

[32] X. Xi, S. Mobayen, H. Ren, and S. Jafari, "Robust finite-time synchronization of a class of chaotic systems via adaptive global sliding mode control," Journal of Vibration and Control, vol. 24, no. 17, pp. 3842-3854, 2017.

[33] E. Campos-Cantón, R. Femat, and G. Chen, "Attractors generated from switching unstable dissipative systems," Chaos: An Interdisciplinary Journal of Nonlinear Science, vol. 22, no. 3, Article ID 033121, 2012.

[34] E.-C. Aguirre-Hernández, J. A. López-Rentería, E. CamposCantón, and C. A. Loredo-Villalobos, "Stability and multiscroll attractors of control systems via the abscissa," Mathematical Problems in Engineering, vol. 2017, Article ID 6743734, 9 pages, 2017.

[35] J. A. López-Rentería, E. Campos-Cantón, B. AguirreHernández, and G. Fernández-Anaya, "A monoparametric family of piecewise linear systems to generate scroll attractors via path-connected set of polynomials," International Journal of Bifurcation and Chaos, vol. 31, no. 3, Article ID 2150034, 2021.

[36] J. R. Pulido-Luna, J. A. López-Rentería, N. R. Cazarez-Castro, and E. Campos, "A two-directional grid multiscroll hidden attractor based on piecewise linear system and its application in pseudo-random bit generator," Integration, vol. 81, pp. 34-42, 2021.

[37] J. R. Pulido-Luna, J. A. López-Rentería, and N. R. CazarezCastro, "Design of a nonhomogeneous nonlinear synchronizer and its implementation in reconfigurable hardware," Mathematical and Computational Applications, vol. 25, no. 3, p. 51, 2020.

[38] J. S. González-Salas, E. Campos-Cantón, F. C. Ordaz-Salazar, and E. Jiménez-López, "Nonlinear filtering preserves chaotic synchronization via master-slave system," Abstract and Applied Analysis, vol. 2013, Article ID 398293, 13 pages, 2013.
[39] G. Chen and T. T. Pham, "Introduction to fuzzy systems," in Applied Mathematics and Nonlinear Science, Chapman Hall/ CRC, Boca Raton, FL, USA, 1st edition, 2005.

[40] V. Santibanez, R. Kelly, and M. A. Llama, "Global asymptotic stability of a tracking sectorial fuzzy controller for robot manipulators," IEEE Transactions on Systems, Man and Cybernetics, Part B (Cybernetics), vol. 34, no. 1, pp. 710-718, 2004.

[41] P. J. Prieto-Entenza, N. R. Cazarez-Castro, L. T. Aguilar, S. L. Cardenas-Maciel, and J. A. López-Rentería, "A Lyapunov analysis for Mamdani type fuzzy-based sliding mode control," IEEE Transactions on Fuzzy Systems, vol. 28, no. 8, pp. 1887-1895, 2020.

[42] E. Euntai Kim, H. Heejin Lee, and M. Mignon Park, "Limitcycle prediction of a fuzzy control system based on describing function method," IEEE Transactions on Fuzzy Systems, vol. 8, no. 1, pp. 11-22, 2000.

[43] E. Campos-Cantón, "Chaotic attractors based on unstable dissipative systems via third-order differential equation," International Journal of Modern Physics C, vol. 27, no. 1, Article ID 1650008, 2016. 